Research Paper

\title{
Combined Use of the Neutrophil-Lymphocyte and Platelet-Lymphocyte Ratios as a Prognostic Predictor in Patients with Operable Soft Tissue Sarcoma
}

\author{
Yao Liang1, $2^{*}$, Wei Wang ${ }^{1,2^{*}}$, Jingjing $\mathrm{Li}^{1,3^{*}}$, Yuanxiang Guan ${ }^{1,2}$, Yi Que1,3, Wei Xiao ${ }^{1,3^{*}}$, Xing Zhang1,3凶 , \\ Zhiwei Zhou ${ }^{1,2} \bowtie$ \\ 1. State Key Laboratory of Oncology in South China, Collaborative Innovation Center for Cancer Medicine, Guangzhou, China; \\ 2. Department of Gastric Surgery, Sun Yat-sen University Cancer Center, Guangzhou, China; \\ 3. Department of Medical Melanoma and Sarcoma, Sun Yat-sen University Cancer Center, Guangzhou, China \\ * These authors contributed equally to this article \\ $\triangle$ Corresponding authors: Xing Zhang, MD, PhD. Address: Sun Yat-sen University Cancer Center, 651 Dongfeng Road East, Guangzhou 510060, P.R. China. \\ Tel: +86-20-87343029; Fax: +86-20-87343775; Email: zhangxing@sysucc.org.cn and Zhiwei Zhou, MD, PhD. Address: Sun Yat-sen University Cancer Center, 651 \\ Dongfeng Road East, Guangzhou 510060, P.R. China. Tel: +86-20-87343626; Fax: +86-20-87343775. Email: zhouzhw@sysucc.org.cn \\ (c) Ivyspring International Publisher. This is an open access article distributed under the terms of the Creative Commons Attribution (CC BY-NC) license \\ (https://creativecommons.org/licenses/by-nc/4.0/). See http://ivyspring.com/terms for full terms and conditions.
}

Received: 2018.01.10; Accepted: 2018.03.31; Published: 2018.05.25

\begin{abstract}
Background: Preoperative neutrophil-to-lymphocyte ratio (NLR) and platelet-to-lymphocyte ratio (PLR) are associated with poor prognosis in soft tissue sarcoma (STS). The aim of the present study is to determine whether the combination of NLR and PLR (CNP) can better predict patient survival after resection for STS.

Methods: We included 310 STS patients in this retrospective study. Preoperative CNP was calculated as follows: patients with both elevated NLR $(>2.51)$ and PLR $(>191.1)$ were given a score of 2; patients showing an increase in one or neither were allocated a score of 1 or 0 , respectively.

Results: Cut-off values of 2.51 and 191.1 were defined as elevated NLR and PLR, respectively. Elevated CNP was significantly associated with older age $(P=0.034)$, larger tumor size $(P=0.025)$, deeper tumor location $(P=0.044)$, higher tumor grade $(P=0.028)$, a more advanced stage according to the American Joint Committee on Cancer (AJCC) $(P=0.005)$, shorter overall survival (OS) $(P=0.000)$ and shorter disease-free survival (DFS) $(P=0.000)$. Multivariate analysis indicated CNP but not NLR or PLR to be an independent prognostic factor for OS and DFS ( $P=0.000$ and $P=0.001$, respectively).
\end{abstract}

Conclusions: Preoperative CNP is associated with tumor progression and can be considered an independent marker of postoperative survival in patients with STS.

Key words: Soft tissue sarcoma, NLR, PLR, Prognostic factor, Survival

\section{Background}

Soft tissue sarcomas (STSs) are relatively rare but aggressive tumors that account for approximately $0.7 \%$ of all cancers and $0.8 \%$ of cancer mortality [1]. STSs are biologically heterogeneous and due to diverse histology, are classified into over 70 subtypes, with every type exhibiting differences in terms of characteristics, treatment and prognosis [2]. Despite advances in multidisciplinary treatment, the probability of local recurrence and metastasis remains high for STS patients, particularly for those with high-grade tumors owing to the rapid clinical progression of the disease [3]. The 5-year relative survival rate for STS is approximately $65 \%$ [4], yet up to $50 \%$ of patients will develop metastases and die from their disease [5]. Therefore, it is important to identify easily obtainable and economically feasible 
clinical tools that can help guide treatment options and enhance the capability of risk stratification.

Growing evidence demonstrates a critical role of the systemic inflammatory response (SIR) in the development and progression of cancer as well as the response to therapy [6, 7]. Accordingly, several inflammation-based prognostic markers, such as C-reactive protein (CRP) [8], the Glasgow Prognostic Score (GPS) [9], and the high-sensitivity modified Glasgow prognostic score (Hs-mGPS) [10], have been proposed as independent predictors of survival in various types of malignancies, including STS [11]. In addition, as important components of SIR, neutrophils, lymphocytes, and platelets are recognized as participating in the proliferation and migration of tumor cells [12-15]. Recently, two studies indicated that the neutrophil-to-lymphocyte ratio (NLR), a representative index derived from inflammatory cells, can serve as a prognostic factor in STS [16, 17]. Similarly, the platelet-to-lymphocyte ratio (PLR), another SIR indicator based on routine blood analysis, has prognostic value for STS [18]. However, the combined use of these two indices for STS prognosis has not yet been assessed.

Therefore, we incorporated both ratios into a novel measure, the CNP (the combination of NLR and PLR) scoring system and evaluated its prognostic value in patients with operable STS. We also assessed whether CNP can provide additional information compared with NLR or PLR alone.

\section{Methods}

\section{Patients}

All STS patients who underwent extensive and radical resection at Sun Yat-sen University Cancer Center (SYSUCC) from October 1999 to August 2013 were carefully reviewed retrospectively. The inclusion criteria were (1) histologically confirmed STS; (2) surviving at least 30 days postoperatively; (3) without any chemotherapy and/or radiotherapy before serum collection; and (4) adequate and available medical record and following-up data. Patients were excluded if they presented with an acute infection or chronic inflammatory disease, malignancy or any other known autoimmune disorder. Patients with recurrence at presentation or those who were referred for additional resection after previously undergoing unplanned excision were excluded. Finally, a total of 310 patients were included in this study.

\section{Clinical Data Collection}

Laboratory data, including quantification of neutrophils, lymphocytes, and platelets, and other basic hematological parameters, were obtained one week prior to surgery. In cases in which patients received more than one serum test, we utilized the most recent test. Clinical data, such as age at diagnosis, gender and histopathological diagnosis, were extracted retrospectively from the medical records. Stage was classified according to the American Joint Committee on Cancer (AJCC) 7th Edition [19], and tumors were graded according to the French Federation of Cancer Centers Sarcoma Group (FNCLCC) grading system [20]. NLR was defined as the absolute neutrophil count divided by the absolute lymphocyte count. PLR was defined as the absolute platelet count divided by the absolute lymphocyte count. Based on receiver operating characteristic (ROC) curves analyses, the optimal cut-off values of NLR and PLR were 2.51 and 191.1, respectively. We combined the two indicators to define the CNP score as follows: patients with both an elevated NLR (>2.51) and PLR (>191.1) were allocated a score of 2; patients with only one elevated value were allocated a CNP score of 1 ; patients without abnormalities were allocated a score of 0 . Scores of 2, 1 and 0 were classified as "high risk," "intermediate risk" and "low risk," respectively.

The authenticity of this article was validated by uploading the key raw data to the Research Data Deposit public platform (www.researchdata.org.cn) with the approval RDD number of RDDA2017000452.

\section{Patient Follow-up}

Patients were followed-up at our independent follow-up program department every 3 to 6 months for the first 2 years after resection and annually thereafter. Follow-up data were available from patient files or by telephone interview with the patients or guardians. The primary end-point of the study was overall survival (OS), which was defined as the time in months between the operation and death from any cause or to the last date of follow-up (May 01, 2017). The secondary end-point was disease-free survival (DFS), which was determined from the date of resection to the date of tumor recurrence or distant metastasis.

\section{Statistical Analysis}

Data are presented as a number (\%). Comparisons between the three CNP groups were performed using the chi-square $\left(\chi^{2}\right)$ test. The Spearman correlation test was conducted to evaluate relationships between NLR and PLR, and NRP, PLR and OS. The optimal cut-off points for NLR and PLR were determined by ROC analysis, and areas under the curve (AUCs) were calculated. The overall cumulative probability of survival was calculated using the Kaplan-Meier method, and differences in 
survival rates were determined using the log-rank test. Prognostic variables associated with OS and DFS that were significant in univariate analyses were selected for multivariate Cox proportional hazards regression analysis using the forward stepwise method. Hazard ratios (HR) estimated from the Cox analysis are reported as relative risks with corresponding $95 \%$ confidence intervals (CIs). P values were derived from two-tailed tests. A p-value less than 0.05 was considered statistically significant. All analyses were performed using SPSS version 20.0 (SPSS Inc., Chicago, IL, USA.).

Table 1. The relationships between CNP and clinicopathological characteristics

\begin{tabular}{|c|c|c|c|c|c|}
\hline Variables & $\begin{array}{l}\text { All } \\
\text { patients }\end{array}$ & $\begin{array}{l}\text { Score 0 } \\
(n=202)\end{array}$ & $\begin{array}{l}\text { Score } \\
1(n=75)\end{array}$ & $\begin{array}{l}\text { Score } \\
2(n=33)\end{array}$ & $p$-value \\
\hline \multicolumn{6}{|l|}{ Gender } \\
\hline Male & $174(56.1)$ & $117(57.9)$ & 43(57.3) & $14(42.4)$ & 0.244 \\
\hline Female & $136(43.9)$ & $85(42.1)$ & $32(42.7)$ & $19(57.6)$ & \\
\hline \multicolumn{6}{|l|}{ Age (years) } \\
\hline$<50$ & $208(67.1)$ & $145(71.8)$ & $46(61.3)$ & $17(51.5)$ & 0.034 \\
\hline$\geq 50$ & $102(32.9)$ & $57(28.2)$ & $29(38.7)$ & $16(48.5)$ & \\
\hline $\begin{array}{l}\text { Performance } \\
\text { status }\end{array}$ & & & & & 0.001 \\
\hline 0 & $237(76.5)$ & $168(83.2)$ & $52(69.3)$ & $17(51.5)$ & \\
\hline$\geq 1$ & $73(23.5)$ & $34(16.8)$ & $23(30.7)$ & $16(45.5)$ & \\
\hline Tumor size $(\mathrm{cm})$ & & & & & 0.025 \\
\hline$<5$ & $143(46.1)$ & $104(51.5)$ & $29(38.7)$ & $10(30.3)$ & \\
\hline$\geq 5$ & $167(53.9)$ & $98(48.5)$ & $46(61.3)$ & $23(69.7)$ & \\
\hline Tumor site & & & & & 0.132 \\
\hline Upper extremity & $35(11.3)$ & $25(12.2)$ & $5(6.7)$ & $5(16.7)$ & \\
\hline Lower extremity & $82(26.5)$ & $62(30.2)$ & $14(18.7)$ & $6(20)$ & \\
\hline Thoracic/trunk & $91(29.4)$ & $62(30.2)$ & $22(29.3)$ & $7(23.3)$ & \\
\hline Intra-abdominal & $48(15.5)$ & $28(13.7)$ & $16(21.3)$ & $4(13.3)$ & \\
\hline Head/neck & $48(15.5)$ & $24(11.7)$ & $16(21.3)$ & $8(26.7)$ & \\
\hline Others & $6(1.9)$ & $4(2.0)$ & $2(2.7)$ & $0(0.0)$ & \\
\hline Tumor depth & & & & & 0.044 \\
\hline Superficial & $127(41.0)$ & $90(45.0)$ & $29(38.6)$ & $8(22.9)$ & \\
\hline Deep & $183(59.0)$ & $110(55.0)$ & $46(61.4)$ & 27(77.1) & \\
\hline Tumor grade & & & & & 0.028 \\
\hline G1 & $82(26.4)$ & $64(31.7)$ & $16(21.3)$ & $2(6.1)$ & \\
\hline G2 & $119(38.4)$ & $77(38.1)$ & $29(38.7)$ & $13(39.4)$ & \\
\hline G3 & $80(25.8)$ & $43(21.3)$ & $23(30.7)$ & $14(42.4)$ & \\
\hline Missing & $29(9.4)$ & $18(8.9)$ & $7(9.3)$ & $4(12.1)$ & \\
\hline AJCC stage & & & & & 0.005 \\
\hline $\mathrm{IA}+\mathrm{IB}$ & $83(26.8)$ & $65(32.2)$ & $16(21.3)$ & $2(6.1)$ & \\
\hline IIA+IIB & $141(45.5)$ & $92(45.5)$ & $31(41.3)$ & $18(54.5)$ & \\
\hline III+IV & $62(20.0)$ & $29(14.4)$ & $23(30.7)$ & $10(30.3)$ & \\
\hline Unknown & $24(7.1)$ & $16(7.9)$ & $5(6.7)$ & $3(9.1)$ & \\
\hline End-point & & & & & 0.000 \\
\hline Alive & $212(68.4)$ & $154(76.2)$ & $50(66.7)$ & $8(24.2)$ & \\
\hline Dead & $98(31.6)$ & $48(23.8)$ & $25(33.3)$ & $25(75.8)$ & \\
\hline Recurrence & & & & & 0.000 \\
\hline YES & $112(36.1)$ & $61(30.2)$ & $28(37.3)$ & $23(69.7)$ & \\
\hline NO & 198(63.9) & $141(69.8)$ & $47(62.7)$ & $10(30.3)$ & \\
\hline Metastasis & & & & & 0.001 \\
\hline YES & 61(19.7) & $31(15.3)$ & $16(21.3)$ & $14(42.4)$ & \\
\hline $\mathrm{NO}$ & $249(80.3)$ & $171(84.7)$ & $59(78.7)$ & $19(57.6)$ & \\
\hline
\end{tabular}

\section{Results}

\section{Patient and Tumor Characteristics}

Among the 310 patients with STS, 136 (43.9\%) were women and 174 (56.1\%) men. The median age was 39 years, with a range of 5-78 years. At a median follow-up period of 88 months (interquartile range, 47.5-119.25 months), 112 (36.1\%) patients had local relapse, 61 (19.7\%) developed disease recurrence, and $98(31.6 \%)$ died.

The primary tumor sites were located in the upper extremities $(n=35)$, lower extremities $(n=82)$, thorax/trunk $(\mathrm{n}=91)$, intra-abdominal $(\mathrm{n}=48)$, and head/neck $(n=48)$. The mean tumor size at diagnosis was $6.6 \mathrm{~cm}$ (1 to 28). The depth of the tumor was superficial in 127 patients and deep in 183 patients. Tumor pathological subtypes included so-called malignant fibrous histiocytoma (MFH) in 78 patients $(25.2 \%)$, fibrosarcoma in $59(19.0 \%)$, synovial sarcoma in $40(12.9 \%)$, and liposarcoma in $37(11.9 \%)$. A total of 82 patients had grade one sarcoma, 119 had grade two, and 80 had grade three. The majority of patients had stage I or II disease (224/310, 72.3\%); 62 (20.0\%) had stage III or stage IV.

A total of 202 patients $(65.2 \%)$ had a score of $0 ; 75$ $(24.2 \%)$ had a score of 1 , and $33(10.6 \%)$ had a score of 2. Adjuvant treatments, including chemotherapy (mostly doxorubicin-based combination chemotherapy) and radiotherapy, were planned according to the tumor stage, doctor's selection and patient's desire. The baseline characteristics of the 310 patients are presented in Table 1 and Table S1.

\section{Relationships between NLR and PLR, and NLR, PLR and OS.}

We examined the association between NLR, PLR, and NLR, PLR and OS. We found a significant negative correlation between serum NLR and OS $(\mathrm{r}=-0.175, \mathrm{P}=0.002)$ (Fig. S1a), and between serum PLR and $\mathrm{OS}(\mathrm{r}=-0.180, \mathrm{P}=0.001)$ (Fig. S1b). In addition, a positive association between NLR and PLR $(r=0.606$, $\mathrm{P}=0.000$ ) was observed (Fig. S1c).

\section{Determination of Optimal Cut-off Values}

ROC analysis and AUC calculations (Table S2) revealed that an NLR value of 2.51 should be considered the recommended cut-off value because it was closest to the maximum joint sensitivity (0.459) and specificity (0.764) in predicting OS (AUC: 0.608, 95\% CI:0.537-0.678, P=0.002). Similarly, the optimal cutoff value for PLR was 191.1 (AUC: $0.614,95 \%$ CI: $0.543-0.686, \mathrm{P}=0.001$ ), with a sensitivity and specificity of 0.306 and 0.929 , respectively. The AUC for CNP was 0.644 (95\% CI: 0.574-0.713, $\mathrm{P}=0.000$ ).

\section{Correlation between CNP and Clinicopathological Characteristics}

Table 1 presents the distribution of the clinical background characteristics of the patients in the three CNP groups. The data showed elevated CNP to be 
a

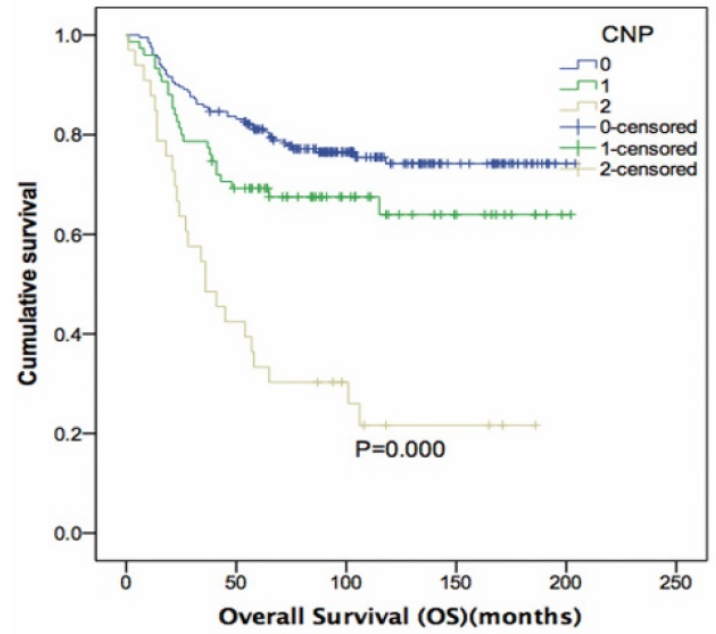

b

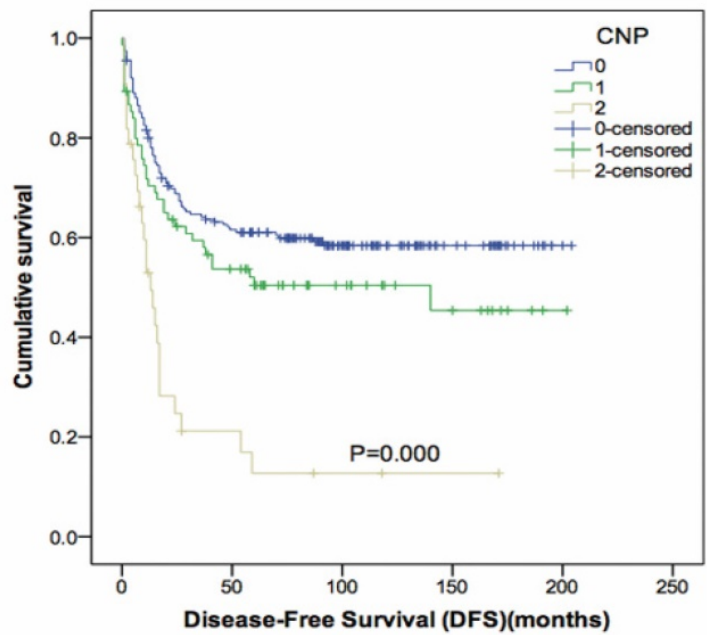

Fig. 1. Kaplan-Meier curves showing overall survival (a) and disease-free survival (b) stratified by CNP.

significantly associated with older age $(\mathrm{P}=0.034)$, larger tumor size $(\mathrm{P}=0.025)$, deeper tumor location $(\mathrm{P}=0.044)$, higher tumor grade $(\mathrm{P}=0.028)$ and more advanced AJCC stage $(\mathrm{P}=0.005)$. No significant differences in gender or tumor site were identified among the three groups $(\mathrm{P}=0.244, \mathrm{P}=0.132$ respectively).

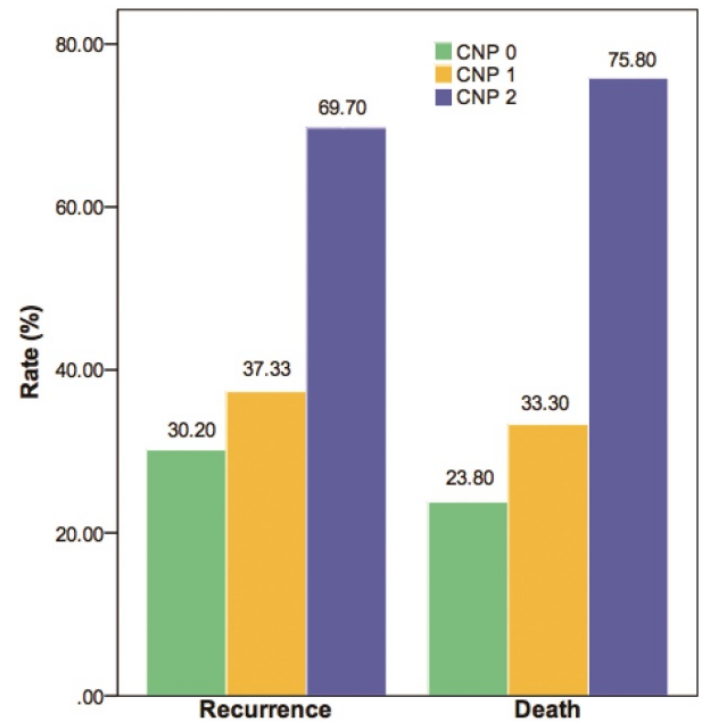

Fig. 2. Recurrence rate and mortality according to CNP.

\section{Survival Analysis}

Kaplan-Meier analysis and the log-rank test showed that high-risk patients had a poorer prognosis than those with intermediate or low risk. An elevated CNP score was significantly associated with shorter OS $(\mathrm{P}=0.000)$ and DFS $(\mathrm{P}=0.000)$ (Fig. 1). The recurrence and death rates of patients in CNP groups 0,1 and 2 differed significantly: 30.20, 37.33 and
$69.70 \%, 23.80,33.30$ and $75.80 \%$, respectively $(\mathrm{P}=0.000$; Fig. 2).

In univariate analyses of OS and DFS, NLR, PLR and CNP were all found to be prognostic factors. Other identified prognostic factors included tumor size, tumor depth, tumor grade and AJCC stage (Table 2).

Table 2. Univariate analyses of DFS and OS in STS patients

\begin{tabular}{|c|c|c|c|c|}
\hline & Disease-free survival & & Overall survival & \\
\hline & HR(95\% CI) & $p$-value & $\mathrm{HR}(95 \% \mathrm{CI})$ & $p$-value \\
\hline Sex & & 0.934 & & 0.190 \\
\hline Male & 1.000 & & 1.000 & \\
\hline Female & $1.014(0.730-1.09)$ & & $1.304(0.877-1.937)$ & \\
\hline Age (years) & & 0.011 & & 0.395 \\
\hline$<50$ & 1.000 & & 1.000 & \\
\hline$\geq 50$ & $1.538(1.103-2.145)$ & & $1.195(0.793-1.802)$ & \\
\hline Tumor size $(\mathrm{cm})$ & & 0.000 & & 0.014 \\
\hline$<5$ & 1.000 & & 1.000 & \\
\hline$\geq 5$ & $2.181(1.537-3.093)$ & & $1.676(1.109-2.535)$ & \\
\hline Tumor depth & & 0.000 & & 0.000 \\
\hline Superficial & 1.000 & & 1.000 & \\
\hline Deep & $3.006(2.043-4.422)$ & & $3.174(1.942-5.190)$ & \\
\hline Tumor grade & & 0.000 & & 0.000 \\
\hline G1 & 1.000 & & 1.000 & \\
\hline G2 & $2.268(1.340-3.837)$ & & $4.339(1.821-10.336)$ & \\
\hline G3 & $5.300(3.132-8.971)$ & & $10.918(4.647-25.652)$ & \\
\hline AJCC stage & & 0.000 & & \\
\hline $\mathrm{IA}+\mathrm{IB}$ & 1.000 & & 1.000 & 0.000 \\
\hline IIA+IIB & $2.294(1.402-3.751)$ & & $4.571(2.065-10.115)$ & \\
\hline III+IV & $5.089(3.014-8.590)$ & & $9.540(4.224-21.544)$ & \\
\hline NLR & & 0.001 & & 0.000 \\
\hline$<2.51$ & 1.000 & & 1.000 & \\
\hline$\geq 2.51$ & $1.778(1.271-2.487)$ & & $2.229(1.497-3.318)$ & \\
\hline PLR & & 0.000 & & 0.000 \\
\hline$<191.1$ & 1(referent) & & 1.000 & \\
\hline$\geq 191.1$ & $2.498(1.688-3.698)$ & & $3.585(2.327-5.522)$ & \\
\hline CNP & & 0.000 & & 0.000 \\
\hline 0 & 1(referent) & & 1.000 & \\
\hline 1 & $1.363(0.924-2.011)$ & & $1.570(0.968-2.547)$ & \\
\hline 2 & $3.333(2.129-5.218)$ & & $4.653(2.863-7.566)$ & \\
\hline $\begin{array}{l}\text { DFS, disease-fre } \\
\text { hazard ratio; CI, } \\
\text { Cancer; NLR, ne } \\
\text { the combination }\end{array}$ & $\begin{array}{l}\text { e survival; OS, overall } \\
\text { confidence interval; } \\
\text { utrophil lymphocyte }\end{array}$ & $\begin{array}{l}\text { urvival; S } \\
\text { grade; AJ } \\
\text { tio; PLR, }\end{array}$ & $\begin{array}{l}\text { 2, Soft tissue sarcoma } \\
\text { C, American Joint Cor } \\
\text { atelet lymphocyte rat }\end{array}$ & $\begin{array}{l}\text { HR, } \\
\text { mittee on } \\
\text {; CNP, } \\
\text { ratio. }\end{array}$ \\
\hline
\end{tabular}


Multivariate analyses using the variables selected from univariate analysis as covariates revealed that preoperative CNP was also an independent prognostic factor for OS and DFS $(\mathrm{P}=0.001$ and $\mathrm{P}=0.000$, respectively). The risk of succumbing to STS for patients with a $\mathrm{CNP}=2$ was $>3$ times higher than that for patients with $\mathrm{CNP}=0$ $(\mathrm{OR}=3.425 ; \mathrm{P}=0.000 ; 95 \% \mathrm{CI}, 2.112-5.640)$. Tumor depth and tumor grade were also identified as independent predictors of DFS and OS, whereas NLR and PLR were not statistically significant (Table 3).

Table 3. Multivariate analyses of DFS and OS in STS patients

\begin{tabular}{|c|c|c|c|c|}
\hline & Disease-free survival & & Overall survival & \\
\hline & $\mathrm{HR}(95 \% \mathrm{CI})$ & $p$-value & $\mathrm{HR}(95 \% \mathrm{CI})$ & $p$-value \\
\hline Age (years) & & 0.017 & & \\
\hline$<50$ & 1.000 & & & \\
\hline$\geq 50$ & $1.513(1.077-2.126)$ & 0.017 & & \\
\hline Tumor depth & & 0.000 & & 0.001 \\
\hline Superficial & 1.000 & & 1.000 & \\
\hline Deep & $2.378(1.606-3.521)$ & 0.000 & $2.241(1.363-3.685)$ & \\
\hline Tumor grade & & 0.000 & & 0.000 \\
\hline G1 & 1.000 & & 1.000 & \\
\hline G2 & $1.717(0.999-2.931)$ & 0.050 & 2.931(1.213-7.079) & 0.017 \\
\hline G3 & $4.085(2.371-7.037)$ & 0.000 & 7.165(3.006-17.976) & 0.000 \\
\hline $\mathrm{CNP}$ & & 0.001 & & 0.000 \\
\hline 0 & 1.000 & & 1.000 & \\
\hline 1 & $1.238(0.837-1.830)$ & 0.285 & $1.396(0.858-2.272)$ & 0.179 \\
\hline 2 & $2.427(1.533-3.841)$ & 0.000 & $3.452(2.112-5.640)$ & 0.000 \\
\hline
\end{tabular}

DFS, disease-free survival; OS, overall survival; STS, Soft tissue sarcomas; HR, hazard ratio; $\mathrm{CI}$, confidence interval; $\mathrm{G}$, grade; $\mathrm{CNP}$, the combination of neutrophil lymphocyte ratio and platelet lymphocyte ratio.

In individual subgroup analysis, we found that OS and DFS were better predictors in patients with intermediate or low risk in the G1/G2 subgroup $(\mathrm{P}=0.000, \mathrm{P}=0.001), \mathrm{G} 3$ subgroup $(\mathrm{P}=0.003, \mathrm{P}=0.009)$ and stage I/II $(\mathrm{P}=0.000, \mathrm{P}=0.000)$ and III/IV $(\mathrm{P}=0.000$, $\mathrm{P}=0.014$ ) subgroups (Fig. 3 ).

\section{Discussion}

Despite recent progress in the identification of molecular and genetic alterations in STS [2, 21, 22], routine prognostic risk assessment of STS patients still relies on the traditional model. These traditional prognostic factors have yet to be improved by the incorporation of novel biomarkers, which might be beneficial for individualized treatment. In the present study, we investigated the prognostic value of NLR and PLR as well as CNP, a novel incorporated prognostic system, for 310 STS patients undergoing resection. To the best of our knowledge, this is the first study to show that CNP is associated with tumor progression and can be considered an independent marker of prognosis for patients with STS.

Cumulative evidence has proven that inflammation is a hallmark of cancer. Indeed, cancer-related inflammation can suppress host antitumor immunity and affect tumor response to therapy $[14,23]$. Moreover, the association between SIR and poor prognosis has been reported for many types of cancer, including STS [11, 24]. Nonetheless, the mechanism by which inflammatory cells influence cancer is multifaceted and remains speculative. For example, tumor cells produce various cytokines, such as granulocyte colony-stimulating factor, tumor necrosis factor (TNF), interleukin (IL)-1, -6 , and -8 and vascular endothelial growth factor (VEGF), which activate and recruit neutrophils to the tumor stroma to promote growth [25]. Neutrophils, in turn, release several mediators to provide a stimulating microenvironment that allows for more aggressive tumor behavior by sustaining cell proliferation and facilitating genomic instability [13, 15]. Furthermore, neutrophils interfere with $\mathrm{T}$ lymphocyte function and promote mutagenesis, rendering some individuals more susceptible to carcinogenesis and future cancer progression [26]. Lymphocytes have a key role in tumor defense by inducing cytotoxic cell death and inhibiting tumor cell proliferation and migration [27], and it has been reported that high lymphocytic infiltration into the tumor stroma is associated with better survival and a superior response to systemic therapy [28]. Thus, NLR, a measure of the relative difference between neutrophil and lymphocyte counts, can serve as a prognostic factor for tumor progression and outcome $[29,30]$.

In addition, the hypercoagulable state has also been correlated with malignancy, accounting for a significant percentage of mortality and morbidity in cancer patients [31]. Among coagulation factors, platelets play an essential role in both inflammation and cancer progression by decreasing apoptosis and increasing angiogenesis, thereby promoting the metastatic potential of tumor cells [32]. In turn, tumor cells release procoagulant molecules to activate platelet activity and induce platelet aggregation, which has been proposed to trigger the development of cancer-associated thrombosis [33]. Numerous studies have reported that a high PLR is a negative predictor of prognosis in solid tumors [18,30].

Regardless, in certain cases, NLR and PLR may not function as tumor-specific markers because hormonotherapy and other inflammatory conditions can be confounding factors. Some authors have suggested that NLR or PLR should be assessed together with other inflammatory markers [34]. In light of the above evidence, we attempted to create a new scoring system by combining both NLR and PLR, termed CNP, which is theoretically more convincing and reflects a mixed prognostic value for predicting survival. 


\section{a}

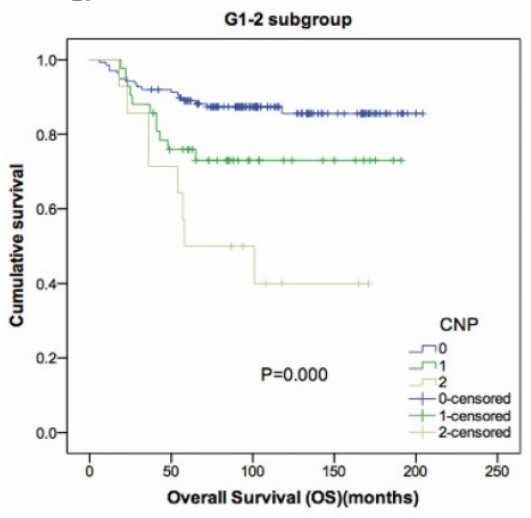

C

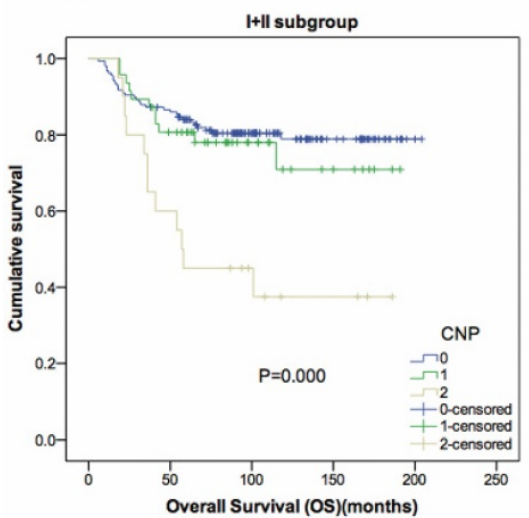

e

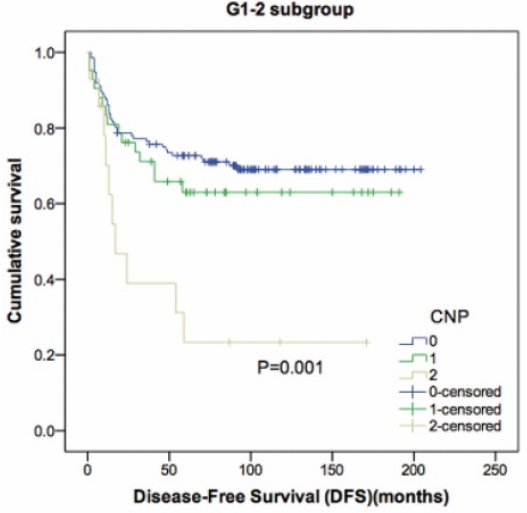

g

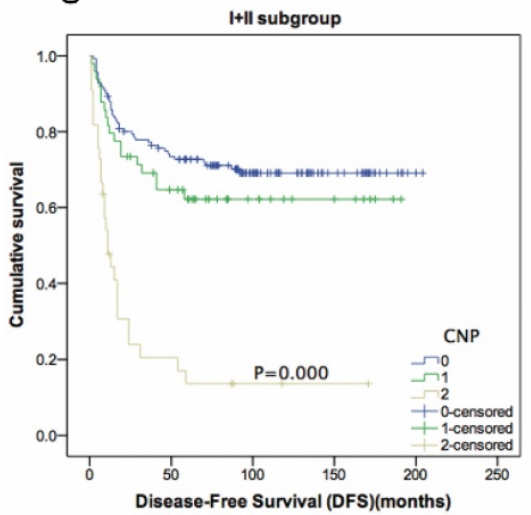

b

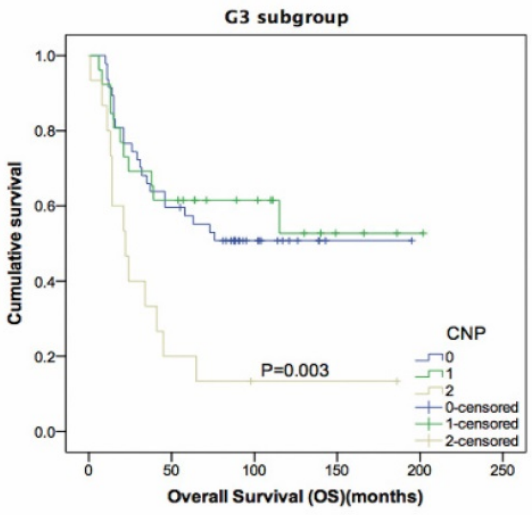

d

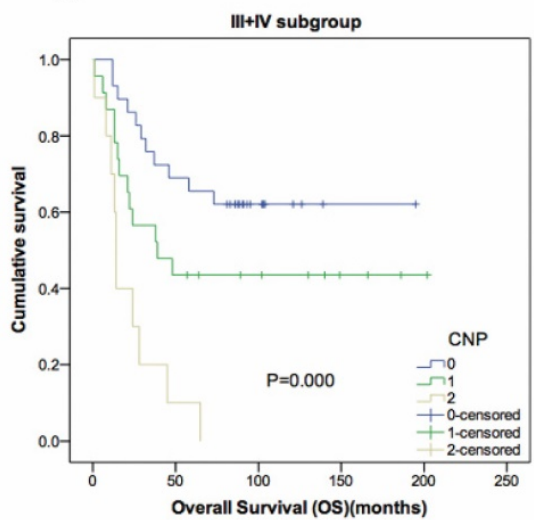

$f$

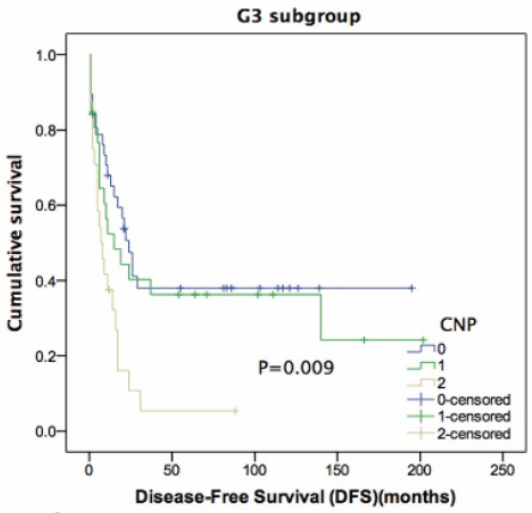

$\mathrm{h}$

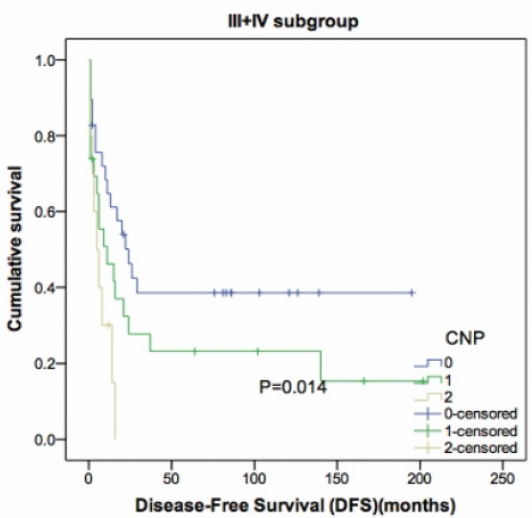

Fig. 3. Kaplan-Meier curves showing overall survival and disease-free survival according to CNP in 310 STS patients. Patients were stratified according to the grade and AJCC stage. (a) Kaplan-Meier analysis of overall survival in G1-2 subgroup. (b) Kaplan-Meier analysis of overall survival in G3 subgroup. (c) Kaplan-Meier analysis of overall survival in I+II subgroup. (d) Kaplan-Meier analysis of overall survival in III+IV subgroup. (e) Kaplan-Meier analysis of disease-free survival in G1-2 subgroup. (f) Kaplan-Meier analysis of disease-free survival in G3 subgroup. (g) Kaplan-Meier analysis of disease-free survival in I+II subgroup. (h) Kaplan-Meier analysis of disease-free survival in III+IV subgroup. 
In our study of 310 STS patients, we identified significant relationships between NLR and PLR and between NLR, PLR and OS; these results suggest that these parameters can serve as biological markers for prognostic estimates. In addition, according to ROC curve analysis, the AUC value of CNP exceeded those of NLR and PLR, for a more convincing conclusion that CNP exhibits comparable prognostic ability that is even stronger than NLR or PLR alone.

Furthermore, the prognosis of patients worsened as the CNP score increased from 0 to 1 to 2 , which is similar to findings reported in patients with hepatocellular carcinoma [35] and gastric cancer [36]. More importantly, we found that patients who had a higher CNP score were more likely to be older in age and have a larger tumor size, a deeper tumor location, a higher tumor grade and a more advanced AJCC stage compared with those with a low CNP score. These findings show that CNP may indicate a more aggressive tumor in STS patients. Cummings et al. [37] also found that an elevated CNP was associated with advanced stage, high tumor burden and metastatic potential in endometrial cancer, thus demonstrating that high CNP significantly parallels tumor progression. Their conclusion was consistent with the findings of our study.

Univariate analysis revealed that all inflammation-based prognostic scores have statistical significance regarding OS and DFS. However, after excluding confounding factors using a Cox regression model of multivariate analysis, we found that only CNP, and not NLR or PLR, was independently associated with OS and DFS. Kaplan-Meier analysis and the log-rank test showed that CNP was able to divide patients into three independent groups. These findings suggest that CNP has a substantial effect and is superior to NLR or PLR as a predictive factor in patients with STS. Although no studies have evaluated simultaneous measurement of these markers in patients with STS, Feng et al. concluded that the combination of NLR and PLR is a significant predictor of OS, with greater value than when used alone in patients with resected esophageal squamous cell carcinoma [38]. Goh et al. produced similar findings in gastrointestinal stromal tumors [34]. Further subgroup analysis also showed a high CNP to be associated with decreased OS and DFS in G1-2, G3, stage I-II and III-IV subgroups. CNP has strong discriminatory power in STS patients with different grades and stages.

Over the last decade, there have been massive efforts to identify clinical biomarkers for predicting outcomes in tumor patients, though expense and technological limitations have precluded their use. Blood-based biomarkers are now recognized to be attractive practical tools, as peripheral blood is easily accessible, routinely tested and relatively noninvasive. Therefore, a blood count-derived measure, CNP has the advantage of being easily measurable, cost-efficient and highly repeatable in clinical practice. Moreover, CNP may constitute a new and valuable tool for custom surveillance schedules and individualized therapeutic strategies for patients of different risk subgroups. Patients with a high preoperative $\mathrm{CNP}$ value may require more intense therapy and more frequent follow-up.

As with all studies, this work has several limitations. First, the study is limited by its retrospective nature and the mixture of various histological types of STS. Second, the sample size collected from a single center was relatively small, and thus it is difficult to perform further verification analysis by separating patients into training and validation cohorts. In addition, information on operational approaches and adjuvant therapies was incomplete, which may have influenced our analysis. Another limitation is the fact that we did not routinely measure and analyze other systemic inflammatory serum markers, such as CRP or Hs-mGPS. Consequently, future studies, particularly prospective multicenter trials with a large cohort, are required to confirm our results and to assess the validity of the prognostic predictors.

\section{Conclusions}

In conclusion, our study provides the first evidence that CNP, a novel and economical inflammation-based prognostic score, is associated with tumor progression and can be considered an independent prognostic factor for predicting OS and DFS in STS patients. Moreover, compared to the established prognostic indices of PLR and NLR, CNP displayed superior prognostic ability in our study population. We recommend the routine measurement of this marker in clinical practice. Nonetheless, larger prospective studies are warranted to confirm these preliminary results and to investigate the underlying mechanisms.

\section{Supplementary Material}

Supplementary figures and tables.

http://www.jcancer.org/v09p2132s1.pdf

\section{Acknowledgments}

Work on this article was supported in part by the National Natural Science Foundation of China (Grant Nos.81572403,81372887) and the National Program on Key Basic Research Project (973 Program) (Grant No.2013CB910500). None of the funding bodies played a role in data collection, analysis, or 
interpretation of data, the writing of the manuscript, or the decision to submit the manuscript for publication.

\section{Availability of data and materials}

The datasets generated and analyzed during the current study are available at the Research Data Deposit public platform (www.researchdata.org.cn) with the approval RDD number of RDDA2017000452.

\section{Ethics approval and consent to participate}

This study was approved by the institutional ethical review board of Sun Yat-sen University Cancer Center. Each author certifies that all investigations were conducted in conformity with the ethical principles. Written informed consent was obtained from all patients or guardians (for minors) included in the study.

\section{Competing Interests}

The authors have declared that no competing interest exists.

\section{References}

1. Siegel RL, Miller KD, Jemal A. Cancer Statistics, 2017. CA: a cancer journal for clinicians. 2017; 67: 7-30.

2. Comprehensive and Integrated Genomic Characterization of Adult Soft Tissue Sarcomas. Cell. 2017; 171: 950-65.

3. Pasquali S, Bonvalot S, Tzanis D, Casali PG, Trama A, Gronchi A. Treatment challenges in and outside a network setting: Soft tissue sarcomas. European journal of surgical oncology: the journal of the European Society of Surgical Oncology and the British Association of Surgical Oncology. 2017.

4. von Mehren M, Randall RL, Benjamin RS, Boles S, Bui MM, Conrad EU, 3rd, et al. Soft Tissue Sarcoma, Version 2.2016, NCCN Clinical Practice Guidelines in Oncology. Journal of the National Comprehensive Cancer Network: JNCCN. 2016; 14: 758-86.

5. Zagars GK, Ballo MT, Pisters PW, Pollock RE, Patel SR, Benjamin RS, et al. Prognostic factors for patients with localized soft-tissue sarcoma treated with conservation surgery and radiation therapy: an analysis of 1225 patients. Cancer. 2003; 97: 2530-43.

6. Laird BJ, Fallon M, Hjermstad MJ, Tuck S, Kaasa S, Klepstad P, et al. Quality of Life in Patients With Advanced Cancer: Differential Association With Performance Status and Systemic Inflammatory Response. Journal of clinical oncology: official journal of the American Society of Clinical Oncology. 2016; 34: 2769-75

7. Dolan RD, Lim J, McSorley ST, Horgan PG, McMillan DC. The role of the systemic inflammatory response in predicting outcomes in patients with operable cancer: Systematic review and meta-analysis. 2017; 7: 1 .

8. Dalpiaz O, Luef T, Seles M, Stotz M, Stojakovic T, Pummer K, et al. Critical evaluation of the potential prognostic value of the pretreatment-derived neutrophil-lymphocyte ratio under consideration of C-reactive protein levels in clear cell renal cell carcinoma. British journal of cancer. 2017; 116: 85-90.

9. McMillan DC. The systemic inflammation-based Glasgow Prognostic Score: a decade of experience in patients with cancer. Cancer treatment reviews. 2013; 39: 534-40.

10. Proctor MJ, Horgan PG, Talwar D, Fletcher CD, Morrison DS, McMillan DC. Optimization of the systemic inflammation-based Glasgow prognostic score: a Glasgow Inflammation Outcome Study. Cancer. 2013; 119: 2325-32.

11. Nakamura T, Matsumine A, Asanuma K, Matsubara T, Sudo A. The value of the high-sensitivity modified Glasgow prognostic score in predicting the survival of patients with a soft-tissue sarcoma. The bone \& joint journal. 2015; 97-b: 847-52.

12. Lal I, Dittus K, Holmes CE. Platelets, coagulation and fibrinolysis in breast cancer progression. Breast cancer research: BCR. 2013; 15: 207.

13. Gregory AD, Houghton AM. Tumor-associated neutrophils: new targets for cancer therapy. Cancer research. 2011; 71: 2411-6.

14. El-Kenawi A, Ruffell B. Inflammation, ROS, and Mutagenesis. Cancer cell. 2017; 32: 727-9.

15. Powell DR, Huttenlocher A. Neutrophils in the Tumor Microenvironment. Trends in immunology. 2016; 37: 41-52.
16. (P035) The Prognostic Value of Pretreatment Peripheral Blood Neutrophil-to-Lymphocyte Ratio in Patients With Soft Tissue Sarcoma. Oncology (Williston Park, NY). 2016; 30 Suppl.

17. Szkandera J, Absenger G, Liegl-Atzwanger B, Pichler M, Stotz M, Samonigg H, et al. Elevated preoperative neutrophil/lymphocyte ratio is associated with poor prognosis in soft-tissue sarcoma patients. British journal of cancer. 2013; 108: 1677-83.

18. Que Y, Qiu H, Li Y, Chen Y, Xiao W, Zhou Z, et al. Preoperative platelet-lymphocyte ratio is superior to neutrophil-lymphocyte ratio as a prognostic factor for soft-tissue sarcoma. BMC cancer. 2015; 15: 648.

19. Edge SB, Compton CC. The American Joint Committee on Cancer: the 7th edition of the AJCC cancer staging manual and the future of TNM. Annals of surgical oncology. 2010; 17: 1471-4.

20. Neuville A, Chibon F, Coindre JM. Grading of soft tissue sarcomas: from histological to molecular assessment. Pathology. 2014; 46: 113-20.

21. Forker L, Gaunt P, Sioletic S, Shenjere P, Potter R, Roberts D, et al. The hypoxia marker CAIX is prognostic in the UK phase III VorteX-Biobank cohort: an important resource for translational research in soft tissue sarcoma. British journal of cancer. 2017.

22. Aggerholm-Pedersen N, Sorensen BS, Overgaard J, Toustrup K, Baerentzen S, Nielsen OS, et al. A prognostic profile of hypoxia-induced genes for localised high-grade soft tissue sarcoma. British journal of cancer. 2016; 115: 1096-104.

23. Mantovani A, Allavena P, Sica A, Balkwill F. Cancer-related inflammation. Nature. 2008; 454: 436-44.

24. Liang Y, Xiao W, Guan YX, Wang W, Chen HY, Fang C, et al. Prognostic value of the C-reactive protein/Albumin Ratio (CAR) in patients with operable soft tissue sarcoma. Oncotarget. 2017; 8: 98135-47.

25. Guo P, Rafii S. Dangerous Liaisons: Deviant Endothelium NOTCHes toward Tumor Metastasis. Cancer cell. 2017; 31: 301-3.

26. Fridlender ZG, Sun J, Kim S, Kapoor V, Cheng G, Ling L, et al. Polarization of tumor-associated neutrophil phenotype by TGF-beta: "N1" versus "N2" TAN. Cancer cell. 2009; 16: 183-94.

27. Fontana R, Bregni M, Cipponi A, Raccosta L, Rainelli C, Maggioni D, et al. Peripheral blood lymphocytes genetically modified to express the self/tumor antigen MAGE-A3 induce antitumor immune responses in cancer patients. Blood. 2009; 113: 1651-60.

28. Solinas C, Ceppi M, Lambertini M, Scartozzi M, Buisseret L, Garaud S, et al. Tumor-infiltrating lymphocytes in patients with HER2-positive breast cancer treated with neoadjuvant chemotherapy plus trastuzumab, lapatinib or their combination: A meta-analysis of randomized controlled trials. Cancer treatment reviews. 2017; 57: 8-15.

29. Chen ZY, Raghav K, Lieu CH, Jiang ZQ, Eng C, Vauthey JN, et al. Cytokine profile and prognostic significance of high neutrophil-lymphocyte ratio in colorectal cancer. British journal of cancer. 2015; 112: 1088-97.

30. Grenader T, Nash S, Adams R, Kaplan R, Fisher D, Maughan T, et al. Derived neutrophil lymphocyte ratio is predictive of survival from intermittent therapy in advanced colorectal cancer: a post hoc analysis of the MRC COIN study. British journal of cancer. 2016; 114: 612-5.

31. Caine GJ, Stonelake PS, Lip GYH, Kehoe ST. The Hypercoagulable State of Malignancy: Pathogenesis and Current Debate. Neoplasia (New York, NY). 2002; 4: 465-73.

32. Bambace NM, Holmes CE. The platelet contribution to cancer progression. Journal of thrombosis and haemostasis: JTH. 2011; 9: 237-49.

33. Lin RJ, Afshar-Kharghan V, Schafer AI. Paraneoplastic thrombocytosis: the secrets of tumor self-promotion. Blood. 2014; 124: 184-7.

34. Goh BK, Chok AY, Allen JC, Jr., Quek R, Teo MC, Chow PK, et al. Blood neutrophil-to-lymphocyte and platelet-to-lymphocyte ratios are independent prognostic factors for surgically resected gastrointestinal stromal tumors. Surgery. 2016; 159: 1146-56.

35. He CB, Lin XJ. Inflammation scores predict the survival of patients with hepatocellular carcinoma who were treated with transarterial chemoembolization and recombinant human type-5 adenovirus H101. 2017; 12: e0174769.

36. Sun $X$, Liu $X$, Liu J, Chen S, Xu D, Li W, et al. Preoperative neutrophil-to-lymphocyte ratio plus platelet-to-lymphocyte ratio in predicting survival for patients with stage I-II gastric cancer. Chinese journal of cancer. 2016; 35: 57.

37. Cummings M, Merone L, Keeble C, Burland L, Grzelinski M, Sutton K, et al. Preoperative neutrophil: lymphocyte and platelet:lymphocyte ratios predict endometrial cancer survival. British journal of cancer. 2015; 113: 311-20.

38. Feng JF, Huang Y, Liu JS. Combination of neutrophil lymphocyte ratio and platelet lymphocyte ratio is a useful predictor of postoperative survival in patients with esophageal squamous cell carcinoma. OncoTargets and therapy. 2013; 6: 1605-12. 